\title{
K2-19, The first K2 muti-planetary system showing TTVs
}

S. C. C. Barros ${ }^{1,2}$, J. M. Almenara ${ }^{3,4}$, O. Demangeon ${ }^{1}$, M. Tsantaki ${ }^{2}$, A. Santerne $^{2}$, D. J. Armstrong ${ }^{5}$, D. Barrado ${ }^{6}$, D. Brown ${ }^{5}$, M. Deleuil ${ }^{1}$, J. Lillo-Box ${ }^{6}$, H. Osborn ${ }^{5}$, D. Pollacco ${ }^{5}$, L. Abe ${ }^{7}$, P. Andre ${ }^{8}$, P. Bendjoya ${ }^{7}$, I. Boisse ${ }^{1}$, A. S. Bonomo ${ }^{9}$, F. Bouchy ${ }^{1}$,

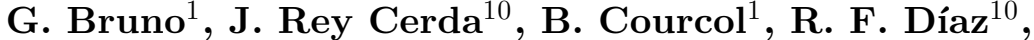
G. Hébrard ${ }^{11,12}$, J. Kirk ${ }^{5}$, J.C. Lachurié ${ }^{8}$, K. W. F. Lam ${ }^{5}$, P. Martinez ${ }^{8}$, J. McCormac ${ }^{5}$, C. Moutou ${ }^{13,1}$, A. Rajpurohit ${ }^{1}$, J.-P. Rivet ${ }^{7}$, J. Spake ${ }^{5}$, O. Suarez ${ }^{7}$, D. Toublanc ${ }^{8,14}$ and S. R. Walker

${ }^{1}$ Aix Marseille Université, CNRS, LAM (Laboratoire d'Astrophysique de Marseille) UMR 7326, 13388, Marseille, France email: susana.barros@astro.up.pt

${ }^{2}$ Instituto de Astrofísica e Ciências do Espaço, Universidade do Porto, CAUP, Rua das Estrelas, PT4150-762 Porto, Portugal

${ }^{3}$ Univ. Grenoble Alpes, IPAG, F-38000 Grenoble, France

${ }^{4}$ CNRS, IPAG, F-38000 Grenoble, France

${ }^{5}$ Department of Physics, University of Warwick, Gibbet Hill Road, Coventry, CV4 7AL, UK

${ }^{6}$ Departamento de Astrofísica, Centro de Astrobiología (CSIC-INTA), ESAC campus 28691 Villanueva de la Cañada (Madrid), Spain

${ }^{7}$ Laboratoire Lagrange, UMR7239, Université de Nice Sophia-Antipolis, CNRS, Observatoire de la Cote d'Azur, F-06300 Nice, France

${ }^{8}$ Observatoire de Belesta en Lauragais - Assoc. Astronomie Adagio 30 Route de Revel 31450 Varennes, France

${ }^{9}$ INAF - Osservatorio Astrofisico di Torino, via Osservatorio 20, 10025, Pino Torinese, Italy

${ }^{10}$ Observatoire Astronomique de l'Universite de Genève, 51 chemin des Maillettes, 1290 Versoix, Switzerland

${ }^{11}$ Institut d'Astrophysique de Paris, UMR7095 CNRS, Université Pierre \& Marie Curie, 98bis boulevard Arago, 75014 Paris, France

${ }^{12}$ Observatoire de Haute-Provence, Université d'Aix-Marseille \& CNRS, 04870 Saint Michel l'Observatoire, France

${ }^{13}$ CNRS, Canada-France-Hawaii Telescope Corporation, 65-1238 Mamalahoa Hwy., Kamuela, HI 96743, USA

${ }^{14}$ Universite de Toulouse, UPS-CNRS, IRAP, 9 Av. colonel Roche, 31028 Toulouse, France

Abstract. In traditional transit timing variations (TTVs) analysis of multi-planetary systems, the individual TTVs are first derived from transit fitting and later modelled using n-body dynamic simulations to constrain planetary masses. We show that fitting simultaneously the transit light curves with the system dynamics (photo-dynamical model) increases the precision of the TTV measurements and helps constrain the system architecture. We exemplify the advantages of applying this photo-dynamical model to a multi-planetary system found in K2 data very close to $3: 2$ mean motion resonance, K2-19. In this case the period of the larger TTV variations (libration period) is much longer ( $>1.5$ years) than the duration of the $\mathrm{K} 2$ observations ( 80 days). However, our method allows to detect the short period TTVs produced by the orbital conjunctions between the planets that in turn permits to uniquely characterise the system. Therefore, our method can be used to constrain the masses of near-resonant systems even when the full libration curve is not observed. 
Keywords. Planet detection, dynamical evolution and stability, K2-19, radial velocity

\section{Introduction}

Transit timing variations (TTVs) are caused by the mutual gravitational interaction of planets which perturb each others' orbit. These are larger when the planets are close to mean-motion resonances (MMR; Escude et al. 2002, Holman et al. 2005, Agol et al. 2005). In near-resonant systems the resonant angles which measure the displacement of the longitude of the conjunction from the periapsis of each planet, circulate (or librate) over a period much longer than the orbital period of the outer planet, called the libration period or super period (Lithwick et al. 2012). Analyses of the long term TTVs libration curve in Kepler transiting multi-planetary systems have allowed to derive dynamic planetary masses and helped confirm the planetary nature of many candidates e.g. Holman et al. (2010), Lissauer et al. (2011), Steffen et al. (2012).

After the failure of two out of four of the reaction wheels of the Kepler satellite the pointing accuracy was severely degraded. Cleaver engineering allowed the continuation of the mission in a new configuration named K2 (Howell et al. 2014). K2 observes 4 fields a year close to the Ecliptic. The short duration of the observations of each field $(\sim 80$ days) does not favour the detection of TTVs amongst planetary candidates discovered in these observations.

K2-19 (EPIC201505350) is a multi-planetary system detected in the K2 Campaign 1 (C1) data by Armstrong et al. (2015). The K2 observations show 2 transiting planets, one with an orbital period $P_{b} \sim 7.92$ days and radius $R_{b}=7.23 \pm 0.60 R_{\oplus}$ and a companion close to the 3:2 MMR with an orbital period $P_{c} \sim 11.91$ days and radius $R_{b}=4.21 \pm$ $0.31 R_{\oplus}$. The closeness to resonance implied that K2-19 was a good candidate for TTV and the brightness of the host star allowed follow-up transit observations from the ground. Approximately 200 days after the end of the K2 C1, a ground based transit was obtained showing TTVs of the inner planet with an amplitude of 1 hour, allowing the authors to validate the system. Here we present a photo-dynamical analysis Carter et al. (2012) of the K2-19 system and allow us to detect TTVs in the K2 data alone.

\section{Method}

\subsection{Observations}

K2-19 was observed during Campaign 1 of the K2 mission between 2014 June 3 and 2014 August 20 spanning $\sim 80$ days. We downloaded the pixel data from the Mikulski Archive for Space Telescopes (MAST) $\dagger$ and used a modified version of the CoRoT imagette pipeline (Barros et al. 2014) to extract the light curve. We corrected the flux dependence with position due to the loss of pointing stability following Barros et al.in prep.

Furthermore, we obtained 10 spectroscopic observations of K2-19 from 2015 February 21 to 2015 April 25 with the SOPHIE spectrograph mounted on the $1.93 \mathrm{~m}$ telescope at the Observatoire de Haute-Provence (Perruchot et al. 2011, Bouchy, et al. 2013). From these we derive radial velocities using a similar method to Santerne et al. (2012). Unfortunately, the radial velocity measurements are not precise enough to detect the stellar reflex velocity due to any of the planets. The spectroscopic observations were also used to derive the host stellar parameters following the methodology described in Tsantaki et al. (2013). We obtained $T_{\text {eff }}=5390 \pm 180 \mathrm{~K}, \log g=4.42 \pm 0.34 \mathrm{dex}, \xi_{t}=$ 
$1.02 \pm 0.24 \mathrm{~km} \mathrm{~s}^{-1}$, and $[\mathrm{Fe} / \mathrm{H}]=0.19 \pm 0.12 \mathrm{dex}$, hence it is a K-dwarf. These were used to derive the stellar mass and radius by interpolating the stellar evolution models using the MCMC described in Díaz et al. (2014) . We obtained $M_{*}=0.918_{-0.070}^{+0.086} \mathrm{M}_{\odot}$ and $R_{*}=0.926_{-0.069}^{+0.19} \mathrm{R}_{\odot}$.

\subsection{Photo-dynamical model}

All the transits and radial velocities were modelled simultaneously with an n-body dynamical integrator that accounts for the gravitational interactions between all components of the system. We use the MERCURY n-body integrator Chambers (1999) to compute the 3 dimensional position and velocity of all system components as a function of time. We assume that only the host star and two planets are present. The stellar velocity projected onto the line-of-sight is used to model the observed radial velocities. To model the transits, we use the Mandel \& Agol (2002) transit model parametrised by the planet-tostar radius ratio, quadratic limb darkening coefficients for each filter and using the sky projection of the planet-star separation computed from the output of MERCURY. This photo-dynamical model is coupled to a Monte Carlo Markov Chain (MCMC) routine, described in detail in Díaz et al. (2014), in order to derive the posterior distribution of the parameters.

To minimise correlations between the model parameters which prevent adequate exploration of the parameter space, we used the Huber et al. (2013) parametrisation. We run 46 independent MCMC chains and combined the results as described by Díaz et al. (2014) . Initially more than 100 chains were started at random points to explore the parameter space. It was found that the chains converged to the same region of the parameter space. To explore this region of the parameter space we launched the 46 independent MCMC chains . The chains were thinned by 970 which was the maximum correlation length of the parameters and merged as described by Díaz et al. (2014) resulting in a final merged chain with 3500 independent points. Further details about the photo-dynamic method can be found in Almenara et al. (2015).

\section{Results}

We find that $\mathrm{K} 2-19 \mathrm{~b}$ has a mass of $41.6 \pm 17 M_{\oplus}$ and radius of $7.34 \pm 0.69 R_{\oplus}$ and $\mathrm{K} 2-19 \mathrm{c}$ has a mass of $22.0_{-4.3}^{+10} \quad M_{\oplus}$ and a radius of $4.52 \pm 0.46 R_{\oplus}$. In particular the dynamic parameters that do not depend on stellar models are well constrained: $q_{+}=$ $\frac{M_{p, b}+M_{p, c}}{M_{\star}}=0.000193_{-0.000031}^{+0.00011}$ and $q_{p}=\frac{M_{p, c}}{M_{p, b}}=0.56 \pm 0.19$.

\subsection{Transit timing variations}

To derive the transit times, we calculate the mid point between the first and fourth transit contact using the MERCURY dynamic model output. Therefore, our transit time measurements include information on the system architecture and dynamics and as such are better constrained than direct measurement of the transit times in the light curve.

We find significant TTVs for the K2 observations. These are shown in Figure 1 where the chopping is clearly visible. So this method will be useful for short duration observations like K2, TESS and CHEOPS.

Armstrong et al. (2015) predicted that the resonant timescale of the system is 5 years and hence it is not detectable with the current observation baseline. Therefore, the phase curve cannot be used to constrain the planetary masses e.g. (Lithwick et al. 2012). However, we detect the short period chopping signal at the much shorter synodic timescale. In our case the detection of the chopping signal at the short synodic timescale 


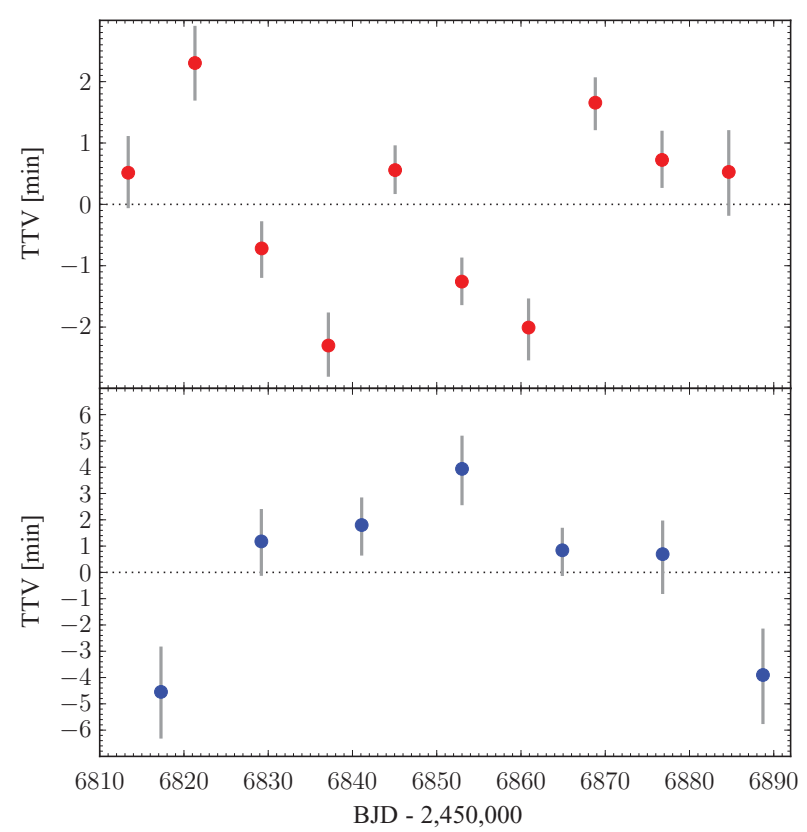

Figure 1. Transit times derived from K2 observations with the photo-dynamic model after removing a linear ephemeris.

allows us to directly determine planetary masses. This can be intuitively understood using the equations derived by Nesvorný \& Vokrouhlický (2014), Deck \& Agol (2015) although our system might not obey their model assumptions. We also find that, as expected, without the detection of the libration period the orbital eccentricities are poorly constrained.

In (Barros et al. 2015) a photodynamic analysis of the K2 data and 3 follow-up transits of planet $\mathrm{b}$ is presented. Our model predicts the times of the follow-up ground based transits to be $2457082.65858_{-0.094}^{+0.076}, 2457090.57608_{-0.10}^{+0.082}, 2457098.49117_{-0.11}^{+0.09}$ respectively for epochs 34, 35 and 36 which are within 1 sigma of the measured values presented in (Barros et al. 2015). Therefore, we conclude that our system solution is robust and it is not significantly affected by spurious TTV due to systematics or spots.

\section{Comparison of photo-dynamic model with traditional TTV computation}

For comparison, we computed the transit times directly from the K2 light curves using a procedure similar to what is described in Barros et al. (2013). For each planet the transits were fitted simultaneously ensuring the same transit shape. In Figure 2, we compare the derived transit times using the photo-dynamic model and the transit times derived with a standard procedure. To compute the ephemeris we use only the values of the observed transit times derived with the photo-dynamic model. For planet b we derived the ephemeris: $T_{b}($ BJD $)=7.921101(69) \times$ Epoch $+2456813.3767(21)$ and for planet c $T_{c}(\mathrm{BJD})=11.90727(58) \times$ Epoch $+2456817.2755(22)$. For each planet the respective and same ephemeris was subtracted from the transit times derived with both methods so that we could directly compare them.

We find that the difference of the transits times for both methods is less than $3 \sigma$ hence the transit times from both methods agree. The higher discrepancy is found for 


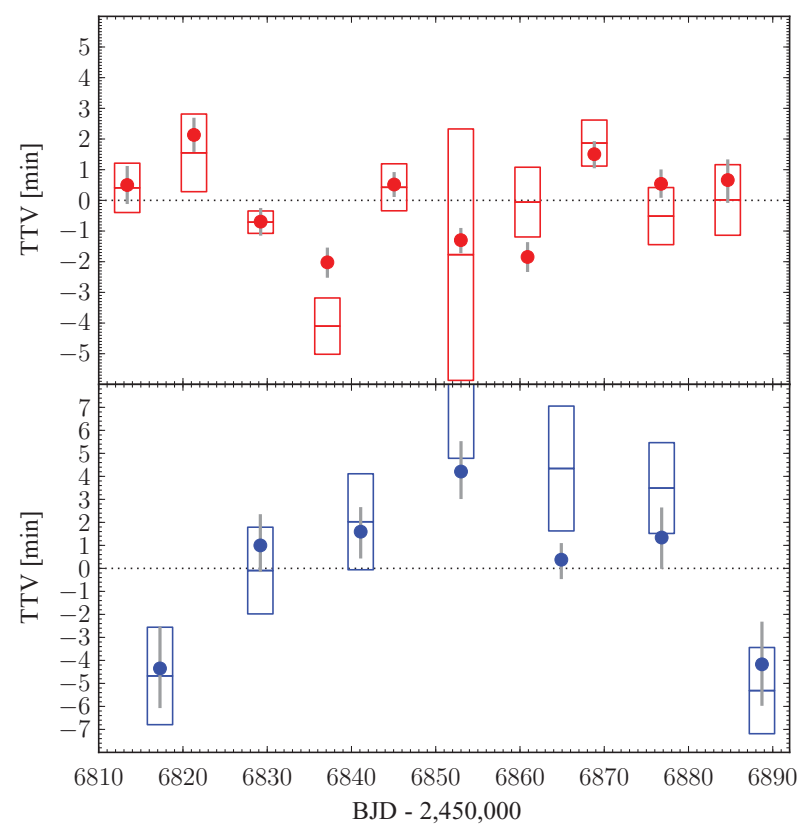

Figure 2. Comparison of the TTVs derived by the photo-dynamic model (as circles) with TTVs derived using a standard transit fitting (as boxes with the size of the 1 sigma error) for planet $b$ (top panel) and planet c (bottom panel). For each planet we use the respective ephemeris derived using the photo-dynamic estimated values of only the observed transits which are marked in red for planet $\mathrm{b}$ and in blue for planet $\mathrm{c}$.

epoch 3 of planet $b$ (the 4th data point in Figure 2). The transit at epoch 3 shows signs of systematic noise. It has been shown by Barros et al. (2013) that, in this case, the errors of the transit times are underestimated, therefore, a difference of $3 \sigma$ is not surprising. Using our photo-dynamic method, we obtain the double of the precision of the transit times as compared to the traditional method that does not include the dynamical constrains. For the K2-19 system the difference increases the significance of the TTVs for planet b and planet c, even in the short duration of the K2 observations, allowing us to better constrain the system architecture. The application of the photo-dynamic method to all available transits of the K2-19 system is presented in Barros et al. 2015 submitted.

\section{Take home messages}

- Applying a photodynamical model leads to a better constrain on the system parameters compared with traditional TTV methods.

- Detecting short period TTVs (chopping) in K2-19 allowed to constrain the system without long time coverage. This will be very important for the analysis of the short duration K2 data and future observations with CHEOPS and TESS.

\section{References}

Agol E., et al. 2005, MNRAS, 359, 567

Almenara J. M. et al. , 2015, MNRAS, in press

Armstrong D. J., et al., 2015a, MNRAS, in press

Barros S. C. C. et al. 2013, MNRAS 430, 3032

Barros S. C. C. et al., 2014, A\& A 569, A74 
Barros S. C. C. et al., 2015, MNRAS 454, 426

Borucki W. J., et al., 2010, Science 327, 977

Bouchy F., et al., 2009, A\&A 505, 853

Bouchy F. et al. 2013, A\&3A549, A49

Carter et al. 2012, Science 337, 556

Chambers J. E., 1999, MNRAS 304, 793

Deck K. M. \& Agol E., 2015, ApJ 802, 116

Díaz R. F., et al. 2014, MNRAS 441, 983

Holman M. J., et al., 2010, Science 1195778

Howell S. B., et al., 2014, PASP 126, 398

Huber D., et al., 2013, Science 342, 331

Lissauer, J. J., Fabrycky, D. C., Ford, E. B., et al. 2011 Nature, 470, 53

Lithwick Y. \& Wu Y., 2012, ApJL 756, L11

Lithwick Y., Xie J., \& Wu Y., 2012, ApJ 761, 122

Mandel K., Agol E., 2002, 580, L171

Nesvorný D. \& Beaugé C., 2010, ApJL ,709, L44

Nesvorný D. \& Vokrouhlický D., 2014,ApJ790, 58

Nesvorný D. et al. 2012, Science 336, 1133

Perruchot S., et al., 2011, in Society of Photo-Optical Instrumentation Engineers (SPIE) Conference Series. p. 15

Santerne A., et al., 2012, A\&A 545, A76

Steffen et al. 2012, MNRAS 421, 2342

Tsantaki M. et al. 2013, A\&A 5 555, A150 